\title{
Charge effect of a liposomal delivery system encapsulating simvastatin to treat experimental ischemic stroke in rats
}

This article was published in the following Dove Press journal:

International Journal of Nanomedicine

29 June 2016

Number of times this article has been viewed

\author{
Mireia Campos-Martorell' \\ Mary Cano-Sarabia ${ }^{2}$ \\ Alba Simats' \\ Mar Hernández-Guillamon' \\ Anna Rosell' \\ Daniel Maspoch 2,3 \\ Joan Montaner ${ }^{1,4}$
}

'Neurovascular Research Laboratory, Institut de Recerca Vall d'Hebron, Universitat Autònoma de Barcelona, Barcelona, ${ }^{2}$ Catalan Institute of Nanoscience and Nanotechnology (ICN2), CSIC and The Barcelona Institute of Science and Technology, Universitat Autònoma de Barcelona, Barcelona, ${ }^{3}$ Institució Catalana de Recerca i Estudis Avançats (ICREA), ${ }^{4}$ Neurovascular Unit, Department of Neurology, Universitat Autònoma de Barcelona, Hospital Vall d'Hebron, Barcelona, Spain
Correspondence: Joan Montaner Neurovascular Research Laboratory, Neurovascular Unit, Institut de Recerca, Hospital Vall d'Hebron, Passeig Vall d'Hebron II 9-129, 08035

Barcelona, Spain

Tel +34934894073

Fax +349348940I5

Email31862jmv@comb.cat
Background and aims: Although the beneficial effects of statins on stroke have been widely demonstrated both in experimental studies and in clinical trials, the aim of this study is to prepare and characterize a new liposomal delivery system that encapsulates simvastatin to improve its delivery into the brain.

Materials and methods: In order to select the optimal liposome lipid composition with the highest capacity to reach the brain, male Wistar rats were submitted to sham or transitory middle cerebral arterial occlusion (MCAOt) surgery and treated (intravenous [IV]) with fluorescentlabeled liposomes with different net surface charges. Ninety minutes after the administration of liposomes, the brain, blood, liver, lungs, spleen, and kidneys were evaluated ex vivo using the Xenogen IVIS ${ }^{\circledR}$ Spectrum imaging system to detect the load of fluorescent liposomes. In a second substudy, simvastatin was assessed upon reaching the brain, comparing free and encapsulated simvastatin (IV) administration. For this purpose, simvastatin levels in brain homogenates from sham or MCAOt rats at 2 hours or 4 hours after receiving the treatment were detected through ultra-high-protein liquid chromatography.

Results: Whereas positively charged liposomes were not detected in brain or plasma 90 minutes after their administration, neutral and negatively charged liposomes were able to reach the brain and accumulate specifically in the infarcted area. Moreover, neutral liposomes exhibited higher bioavailability in plasma 4 hours after being administered. The detection of simvastatin by ultra-high-protein liquid chromatography confirmed its ability to cross the blood-brain barrier, when administered either as a free drug or encapsulated into liposomes.

Conclusion: This study confirms that liposome charge is critical to promote its accumulation in the brain infarct after MCAOt. Furthermore, simvastatin can be delivered after being encapsulated. Thus, simvastatin encapsulation might be a promising strategy to ensure that the drug reaches the brain, while increasing its bioavailability and reducing possible side effects.

Keywords: simvastatin, liposomes, delivery, brain, stroke, rat

\section{Introduction}

Despite all efforts of neuroscience community, stroke still remains a major cause of death and disability worldwide that contributes to the rising costs of health care. ${ }^{1}$ Nowadays, thrombolytic treatment with the tissue plasminogen activator agent is the only existing pharmacological therapy for the acute phase of stroke. ${ }^{2}$ Thus, the need to find efficient neuroprotectant drugs with minimal side effects is becoming an urgency.

Many studies have shown the beneficial effects of simvastatin statin known as 3-hydroxy-3-methylglutaryl coenzyme A reductase inhibitors when administered before or after ischemic stroke in animals as well as in decreasing the incidence of stroke 
in patients. ${ }^{3-6}$ The benefits of statins in cerebral ischemia have not been attributed to their cholesterol-lowering therapeutic actions. Alternatively, pleiotropic effects seem to be implicated in their protective role in stroke. ${ }^{7,8}$ Nevertheless, highdose statin-based therapy in humans has been associated with infrequent but undesirable side effects such as myopathy, rhabdomyolysis, and elevated hepatic transaminases. ${ }^{9}$ In this sense, the use of drug delivery systems such as liposomes is being seriously considered in order to reduce the side effects by site-selected delivery of the drug. ${ }^{10}$ In spite of simvastatin being able to cross the blood-brain barrier (BBB) per se due to its lipophilic condition and its molecular weight (418 kDa), it exhibits poor bioavailability. ${ }^{11}$ Furthermore, it has been reported that BBB disruption occurs at an early stage after cerebral occlusion, allowing macromolecules to pass through the spaces between capillary endothelial cells. ${ }^{12}$ Accordingly, nanoparticles might also be able to leak into brain parenchyma during the acute phase of cerebral ischemia. Interestingly, Sahagun et $\mathrm{al}^{13}$ suggested that both size and charge of drug carriers are important in determining net brain permeation.

Here, our hypothesis is that the bioavailability of simvastatin can be enhanced when it is encapsulated into liposomes, optimizing the opportunities to reach the ischemic brain and increasing the neuroprotective effect, while making the drug safer. To this end, in this study, we explored and characterized the tissue internalization patterns of liposomes presenting different net surface charges. From this study, we assessed how simvastatin encapsulated into neutral liposomes is the best candidate to reach the rat ischemic brain compared to the administration of the free drug.

\section{Materials and methods}

\section{Ethics statement}

All procedures were approved by the Ethics Committee for Animal Experimentation of the Vall d'Hebron Research Institute (protocol numbers 01/13 and 58/13) and conducted in compliance with the Spanish legislation and in accordance with the Directives of the European Union. In all experiments, male Wistar rats (270-300 g; Charles River Laboratories Inc., Wilmington, MA, USA) were used. Rats were kept in a climate-controlled environment on a 12-hour light/ 12-hour dark cycle. Food and water were available ad libitum, and analgesia (buprenorphine $0.05 \mathrm{mg} / \mathrm{kg}$ subcutaneous (sc); Divasa Farma-Vic S.A., Barcelona, Spain) was given to all rats before starting the surgical procedure to minimize their pain and discomfort.

\section{Intraluminal transitory middle cerebral arterial occlusion}

Regional cerebral blood flow was monitored during the surgery with a laser Doppler probe (Moor Instruments, Devon, $\mathrm{UK}$ ), and to that end, cranial trepanation was performed the day before middle cerebral arterial occlusion (MCAO) surgery. In summary, animals under isoflurane (Abbott Laboratories, Abbott Park, IL, USA) effects were placed in a stereotactic table, and using a drill of $1 \mathrm{~mm}$ in diameter, a hole was made in the skull, above the region irrigated by middle cerebral artery (MCA). ${ }^{14}$

Infarction in the territory of the MCA was induced by introducing an intraluminal filament, as described previously. ${ }^{14}$ Animals were anesthetized under spontaneous respiration with isoflurane in oxygen during surgery, and body temperature was maintained at $37^{\circ} \mathrm{C}$ using a heating pad connected to a rectal probe. In brief, after surgical exposure of the bifurcation of the external carotid artery and the internal carotid artery on the right side, a silicone-coated nylon monofilament (Doccol Corporation, Sharon, MA, USA, reference number: 403723PK10) was introduced to occlude the MCA. After occlusion, animals were allowed to recover from anesthesia. Reperfusion was induced 90 minutes later, and to this end, animals were re-anesthetized.

Only animals that exhibited a reduction of $>75 \%$ in regional cerebral blood flow after filament placement and a recovery of $>75 \%$ after filament removal were included in the study. Sham-operated rats were submitted to the same experimental surgery without filament insertion, whereas naïve rats did not receive any surgical procedure. Analgesia was administered just after cranial trepanation and also after MCAO or sham surgery.

\section{Experimental design}

A total of 103 animals were needed to complete the study; 45 animals were used to characterize different liposome lipid compositions and 58 were used to evaluate brain simvastatin distribution through ultra-high-protein liquid chromatography (UHPLC). A flow chart has been designed in order to justify the excluded animals and the criteria established (Figure 1).

\section{Neurological evaluation}

Rats were assessed using a nine-point neurological deficit scale (modified Bederson test), as previously described. ${ }^{15}$ Four consecutive tests were conducted: 1$)$ spontaneous activity (moving and exploring $=0$, moving without exploring $=1$, 


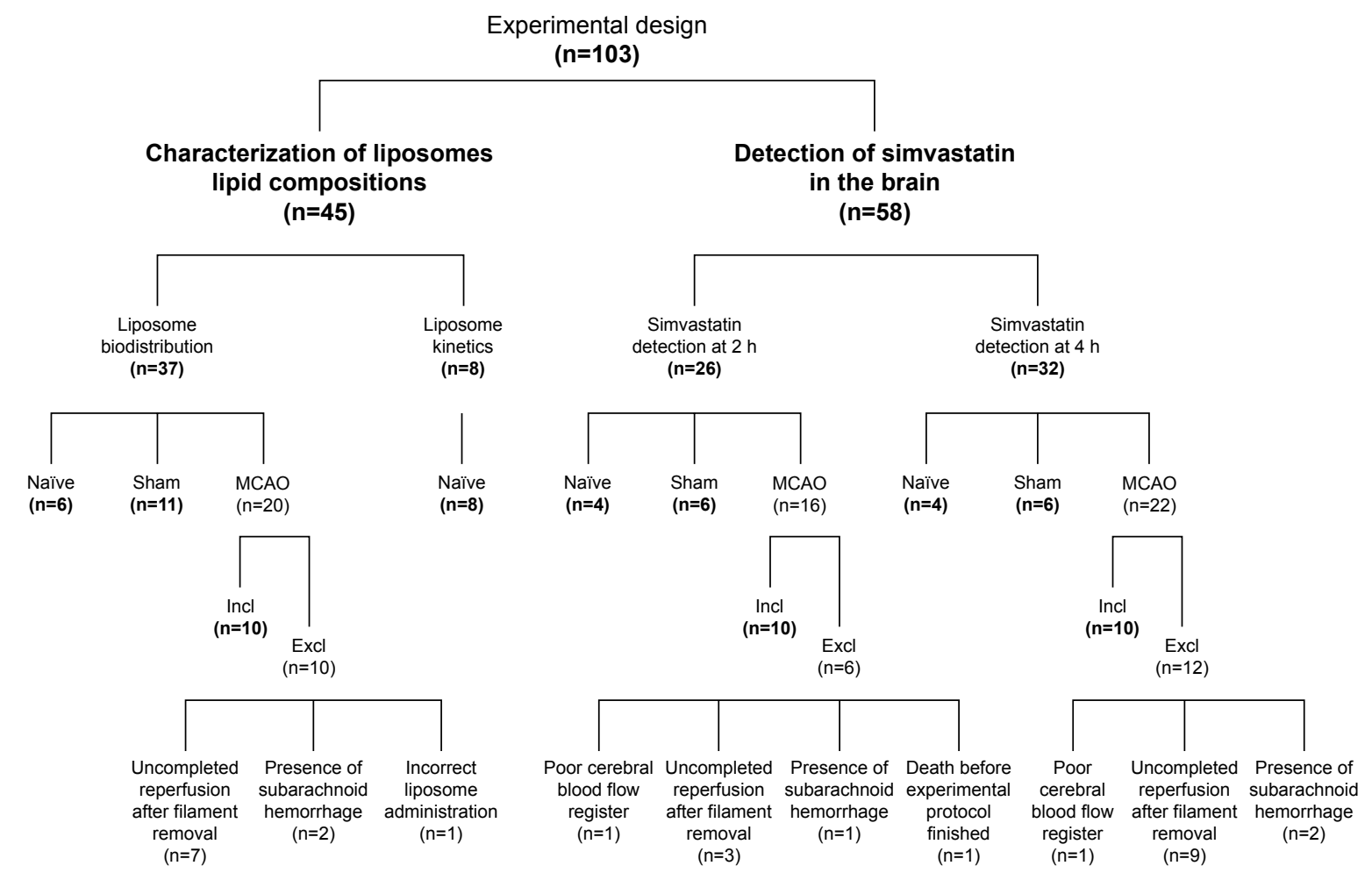

Figure I Diagram summarizing the number of animals included (Incl) and excluded (Excl) per substudy. Abbreviations: MCAO, middle cerebral arterial occlusion; $h$, hours.

no moving or moving only when pulled by the tail $=2$ ); 2) left drifting during displacement (none $=0$, drifting only when elevated by the tail and pushed or pulled $=1$, spontaneous drifting $=2$, circling without displacement or spinning $=3$ ); 3 ) parachute reflex (symmetrical $=0$, asymmetrical $=1$, contralateral $[\mathrm{CL}]$ forelimb retracted $=2)$; and 4) resistance to left forepaw stretching (stretching not allowed $=0$, stretching allowed after some attempts $=1$, no resistance $=2$ ). Neurological score was assessed in a blinded manner at 90 minutes. Scores $>3$ enabled us to ensure that animals were infarcted.

\section{Simvastatin activation}

In all experiments, simvastatin was used in its activated form. Simvastatin is a lactone prodrug that is activated by opening the lactone ring to its $\beta$-hydroxy acid form by hydrolysis in a strong alkaline medium. ${ }^{16}$ For activation, simvastatin was first dissolved in vehicle (distilled $\mathrm{H}_{2} \mathrm{O}$ [75\%], absolute ethanol [10\%], and $0.1 \mathrm{M} \mathrm{NaOH} \mathrm{[15 \% ]).} \mathrm{Then,} \mathrm{the} \mathrm{solution}$ was incubated at $50^{\circ} \mathrm{C}$ for 2 hours adjusting the $\mathrm{pH}$ at 7.2 . To verify the activation of simvastatin, Fourier transform infrared spectroscopy analysis was used. In this study, we focused on examining the frequency range from $3,000 \mathrm{~cm}^{-1}$ to $3,700 \mathrm{~cm}^{-1}$ to verify the formation of multiple hydroxyl groups in the active simvastatin form.

\section{Liposome formulation and characterization}

1,2-Didodecanoyl-sn-glycero-3-phosphocholine (DLPC) was purchased from Lipoid, Steinhausen, Switzerland. Cholesterol, cholesteryl-polyethylene glycol 600 sebacate (CHOL-PEG), and 1,2-dioleoyl-sn-glycero-3-phosphoric acid monosodium salt (DOPA-) were purchased from Sigma Aldrich Co. (St Louis, MO, USA). Cholesteryl $3 \beta-N$ (dimethylaminoethyl) carbamate hydrochloride (CHOL+) and 1,2-dioleoyl-sn-glycero-3-phosphoethanolamine (DOPE) were supplied by Avanti Polar Lipids (Alabaster, AL, USA). Alexa Fluor 750 (AF750) in its succinimidyl ester form was purchased from Thermo Fisher Scientific (Waltham, MA, USA).

Differently charged liposomes (positive, negative, and neutral) were prepared using the thin film hydration method with some modifications. ${ }^{17,18}$ Briefly, DLPC, CHOL, CHOL+, CHOL-PEG, and DOPA were dissolved in chloroform solutions $(100 \mathrm{mg} / \mathrm{mL})$ and mixed at the desired molar ratios under sterile conditions (Table 1). The final concentration 
Table I Liposome composition and characterization

\begin{tabular}{llllll}
\hline Sample & Liposome lipid composition & Encapsulated & $\zeta$ potential (mV) & Mean size (nm) & PDI \\
\hline LIPI+ & DLPC 50\%/CHOL 10\%/CHOL-PEG 5\%/CHOL (+) 35\% & PBS & +30.17 & 157.57 & 0.12 \\
LIP2= & DLPC 50\%/CHOL 45\%/CHOL-PEG 5\% & PBS & +2.21 & 171.07 & 0.10 \\
LIP3- & DLPC 50\%/CHOL 45\%/CHOL-PEG 5\%/DOPA (-) 35\% & PBS & -38.30 & 154.60 \\
LIP4S & DLPC 50\%/CHOL 45\%/CHOL-PEG 5\% & Simvastatin 6 mg/mL & -1.01 & 151.85 & 0.19 \\
\hline
\end{tabular}

Abbreviations: PDI, polydispersity index; DLPC, I,2-didodecanoyl-sn-glycero-3-phosphocholine; CHOL, cholesterol; CHOL-PEG, cholesteryl-polyethylene glycol 600 sebacate; PBS, phosphate-buffered saline; DOPA, I,2-dioleoyl-sn-glycero-3-phosphoric acid monosodium salt.

of lipid was $28 \mathrm{mM}$. The organic solvent was then evaporated under vacuum and nitrogen to obtain a dry lipid film. Afterward, the film was hydrated with the appropriate buffer (phosphate-buffered saline or $6 \mathrm{mg} / \mathrm{mL}$ solution of active simvastatin in water). The resulting lipid suspension was then vigorously shaken, and the liposomes obtained were homogenized by means of an extruder (Lipex Biomembranes, Vancouver, BC, Canada) through a polycarbonate membrane (200 nm pore size) to finally obtain small unilamellar vesicles (SUVs).

Simvastatin encapsulation efficiency was calculated according to the equation $\mathrm{EE}(\%)=\left[\left(C_{\text {total }}-C_{\text {free }}\right) / C_{\text {total }}\right] \times 100$, where $C_{\text {total }}$ is the initial simvastatin concentration and $C_{\text {free }}$ is the concentration of nonencapsulated simvastatin. To quantify $C_{\text {free }}$, liposomes were centrifuged and aliquots of supernatant were analyzed by UV-vis spectroscopy using a Nanodrop ND-1000 (Thermo Fisher Scientific). Mean absorbance values were introduced in a standard calibration curve for the estimation of $C_{\text {free }}$ simvastatin. Liposome integrity was checked by dynamic light scattering and cryogenic transmission electron microscopy (JEOL-JEM 1400 microscope; JEOL, Tokyo, Japan). Particle size distributions by dynamic light scattering and the superficial charge of liposomes - expressed as zeta potential $(\zeta)$ - were measured using Malvern Zetasizer (Malvern Instruments, Malvern, UK) in undiluted samples. The value of zeta potential of simvastatin-loaded liposomes was measured after removing the nonencapsulated simvastatin from preparations by ultracentrifugation at $110,000 \times \mathrm{g}$ for 30 minutes at $10^{\circ} \mathrm{C}$.

\section{Liposome biodistribution}

To monitor distribution in the rat body, liposomes were fluorescently labeled with AF750 (catalog no: A-20011; Thermo Fisher Scientific). To this end, AF750 succinimidyl ester and DOPE- $\mathrm{NH}_{2}$ were conjugated as previously described. ${ }^{19}$ Only conjugated AF750 was detected by thinlayer chromatography $\left(R_{\mathrm{f}}=0.6\right)$, indicating that conjugation was complete. The fluorescent-labeled AF750 liposomes were prepared by incorporating AF750-DOPE into the lipid mixture $(0.38 \mathrm{mM})$.
One milliliter of AF-labeled liposomes (neutral, positively, or negatively charged) was intravenously injected into MCAO ( $n=3-4$ per liposome charge) or sham ( $n=3-4$ per group) rats 90 minutes after ischemia induction and sham surgery, respectively. In MCAO animals, the filament was also removed after 90 minutes of occlusion. Thus, labeled liposomes were administered in ischemic rats just when the reperfusion started. All rats were euthanized 90 minutes after liposome administration, and the brain, liver, spleen, kidneys, lungs, and plasma were obtained from all animals. All organs were entirely maintained on ice until ex vivo analysis with an imaging system (Xenogen IVIS ${ }^{\circledR}$ Spectrum; PerkinElmer Inc., Waltham, MA, USA, located at the Molecular Imaging Platform [PIM] of the CIBER-BBN-VHIR In Vivo Experimental Platform). Brains were analyzed considering both as the entire organ and after slicing them into $2 \mathrm{~mm}$ thick coronal sections in order to explore the exact location of the liposomes. Captured images were then analyzed using the Living Image 4.3.1 software (PerkinElmer Inc.). Liposome signal data were in all cases corrected by subtracting the signal detected in animals free of liposome administration (background). Fluorescent signal data from all tissues were obtained considering the mean value between the anterior and the posterior view. All data obtained by Xenogen IVIS $^{\circledR}$ Spectrum experiments are expressed as radiant efficiency, considered a calibrated measurement of the photon emission from the subject and technically defined as fluorescence emission radiance per incident excitation intensity: photons $/ \mathrm{s} / \mathrm{cm}^{2} / \mathrm{sr}$ (steradian) $/ \mu \mathrm{W} / \mathrm{cm}^{2}$.

\section{Liposome kinetics in plasma}

Naïve rats intravenously received $1 \mathrm{~mL}$ of AF-labeled liposomes neutral or negatively charged ( $n=3$, respectively), whereas positively charged liposomes were discarded in this experiment due to the lack of results obtained in the previous ones. Blood samples were collected from the tail vein after 1.5 hours, 4 hours, or 24 hours of liposomes administration and stored in ethylenediaminetetraacetic acid tubes. Afterward, $50 \mu \mathrm{L}$ of plasma was placed in 96-well plates and analyzed with the Xenogen IVIS ${ }^{\circledR}$ Spectrum. The fluorescent 
signal was quantified with the Living Image software, and the results were expressed as radiant efficiency.

\section{Determination of simvastatin in brain tissue (UHPLC)}

The aim of this substudy was to explore the ability of simvastatin to cross the BBB and assess its possible increased accumulation after its encapsulation into liposomes. Sham or MCAO rats were given $1 \mathrm{~mL}$ of simvastatin through the tail vein, either free or encapsulated into neutral liposomes (previously chosen in the former substudy), and euthanized at 2 hours ( $n=16)$ or 4 hours $(n=16)$ after intravenous (IV) drug administration. Naïve animals $(\mathrm{n}=4$ in each group time) without receiving treatment were also evaluated to correct the background signal. Briefly, under anesthesia, animals were transcardially perfused with a heparin solution in saline followed by pure saline solution, both injected with an infusion pump. Perfusion took 20 minutes and ensured a complete removal of both cerebral blood and circulating simvastatin. Then, brains were removed, divided into ipsilateral (IP) and CL hemispheres, and weighed. Each hemisphere was homogenated separately with a buffer composed of methanol and distilled water (1:1 volume, $5 \mathrm{~mL} / \mathrm{g}$ of tissue) and sonicated. Homogenate supernatants were stored at $-80^{\circ} \mathrm{C}$ until the analysis was performed in all samples at once.

Simvastatin extraction was performed as previously described. ${ }^{20}$ One hundred microliters of each sample of brain homogenate was mixed with $100 \mu \mathrm{L}$ of internal standard (IS) solution $\left(\left[{ }^{2} \mathrm{H}_{6}\right]\right.$-simvastatin hydroxy acid ammonium salt, catalog no: c3520; Alsachim, Illkirch-Graffenstaden, France), which was used to correct simvastatin quantification, and $150 \mu \mathrm{L}$ of acetonitrile. Then, the mixture was vortexed at $153 \times g$ for 30 seconds, and $50 \mu \mathrm{L}$ of $5 \mathrm{M}$ ammonium formate buffer $(\mathrm{pH} 4)$ was added. Samples were vortexed again and centrifuged at $13,000 \times g$ at $4^{\circ} \mathrm{C}$ for 10 minutes. One hundred microliters of supernatant was transferred into the injection tube, and the resulting mixture was diluted with $100 \mu \mathrm{L}$ of mobile phase $(0.5 \mathrm{mM}$ pH 4.5 ammonium acetate:acetonitrile) (70:30). Tubes were vortexed one more time before the samples were injected.

Chromatographic detection was performed using an Acquity UPLC ${ }^{\circledR}$ System (Waters Corporation, Milford, MA, USA) (High Technology Unit [UAT]), with ACQbinary solvent manager and CQ-sample manager. Separation was done in an ACQUITY BEH $2.1 \times 100 \mathrm{~mm}$ phenyl analytical column with precolumn filter. The mobile phase was composed of a mixture of acetonitrile and $0.5 \mathrm{mM}$ ammonium acetate buffer $\mathrm{pH} 4.5$ (30:70) using a gradient elution. Within 1.5 minutes, the concentration was changed to
$30 \%$ of ammonium acetate buffer and subsequently to $5 \%$ of the buffer within 5.25 minutes. Flow rate was $0.25 \mathrm{~mL} / \mathrm{min}$. The analytical column was kept at $35^{\circ} \mathrm{C}$ by column oven, and the solutions were stored in the autosampler at $4{ }^{\circ} \mathrm{C}$. The full loop injection mode was set up to inject $5 \mu \mathrm{L}$ using a $10 \mu \mathrm{L}$ injection loop. Acetonitrile was used as the strong wash solvent, and $70 \%$ ammonium acetate buffer in acetonitrile was used as the weak wash solvent.

Final detection was performed by a tandem mass spectrometry (MS/MS) triple quadrupole system Xevo TQ MS (Waters Corporation) with electrospray ionization in negative mode for simvastatin acid and simvastatin acid- $d_{6}$ (IS) and in positive mode for simvastatin, using the following transitions: 1) $\mathrm{m} / \mathrm{z}=435$ to 114.7 and 318 for simvastatin acid, 2) $\mathrm{m} / \mathrm{z}=441$ to 319.9 for IS, and 3) $\mathrm{m} / \mathrm{z}=419$ to 199 for simvastatin. The range of simvastatin acid quantification was from $4 \mathrm{ng} / \mathrm{mL}$ to $40 \mathrm{ng} / \mathrm{mL}$ in brain homogenate.

\section{Statistical analysis}

Data were analyzed using the GraphPad Prism_v5 software (GraphPad Software, Inc., La Jolla, CA, USA). Statistical significance for intergroup differences was tested by the Student's $t$-test or the analysis of variance followed by the Bonferroni post hoc test for parametric data. For nonparametric data, the Mann-Whitney or Kruskal-Wallis test followed by Dunn's multiple comparison was performed. For parametric data, bars or symbols represent mean $\pm \mathrm{SD}$, and box plots or symbols represent median (interquartile range) for nonparametric data. Two-way analysis of variance was used to compare the liposome signal in plasma over time. A $P$-value $<0.05$ was considered statistically significant at a $95 \%$ confidence level.

\section{Results Liposome characterization}

Series of liposomal formulations with different lipid membrane compositions and net surface charges were initially prepared to determine the best formulation that allows an optimal permeation to the ischemic brain. Table 1 summarizes the characteristics of all liposomes used in this study. Three lipid mixtures were studied: 1) LIP1+, consisting of the cationic lipid mixture DLPC/CHOL/CHOL-PEG/CHOL (+); 2) $\mathrm{LIP} 2=$, formed by the neutral mixture $\mathrm{DLPC} / \mathrm{CHOL} /$ CHOL-PEG; and 3) LIP3-, formed by the anionic lipid mixture DLPC/CHOL/CHOL-PEG/DOPA (-) (Figure 2A-C). In all cases, 5\% of CHOL-PEG was used as PEGylating agent to yield stealth vesicles, and fluorescent AF750 dye was added to the lipid mixture to obtain fluorescent-labeled liposomes for its in vivo tracking. ${ }^{21}$ All formulations consisted 

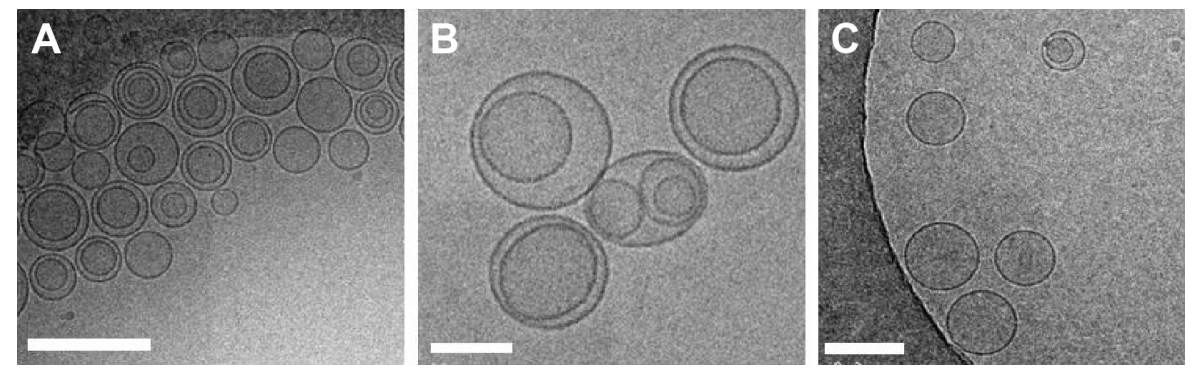

Figure 2 CryoTEM images of liposomes formed by the lipid mixtures.

Notes: (A) DLPC/CHOL/CHOL-PEG/CHOL (+), (B) DLPC/CHOL/CHOL-PEG (=), and (C) DLPC/CHOL/CHOL-PEG/DOPA (-). Scale bar: 200 nm.

Abbreviations: CryoTEM, cryogenic transmission electron microscopy; DLPC, I,2-didodecanoyl-sn-glycero-3-phosphocholine; CHOL, cholesterol; CHOL-PEG, cholesterylpolyethylene glycol 600 sebacate; DOPA, 1,2-dioleoyl-sn-glycero-3-phosphoric acid monosodium salt.

of small multivesicular vesicles (SUVs) with a mean particle size ranging from $154.60 \mathrm{~nm}$ to $171.07 \mathrm{~nm}$.

\section{Cerebral distribution of liposomes}

AF750-labeled liposomes were detected in the whole brain 90 minutes after their administration both in sham and in MCAO animals only when liposomes had neutral or negative zeta potential (Figure 3). A significant signal increase in brain from sham animals was observed only when neutral liposomes administration was compared with the positive ones (neutral: $3.825 \mathrm{e}+008$ [2.960e+008, 6.150e+008] vs positive: $3.860 \mathrm{e}+007$ [0.0, 7.510e+007], $P<0.05$ ) (Figure 3C). Negative liposomes also showed a higher signal than positive ones (negative: $3.05 \mathrm{e}+008$ [2.11e+008, 3.25e+008]), although nonsignificantly. In these brains, neutral and negative liposome fluorescence was associated with areas of high vascular density corresponding with the zone of venous sinuses confluence (Figure 3A).

In accordance, ischemic rats administered with neutral liposomes also presented a significant signal increase in

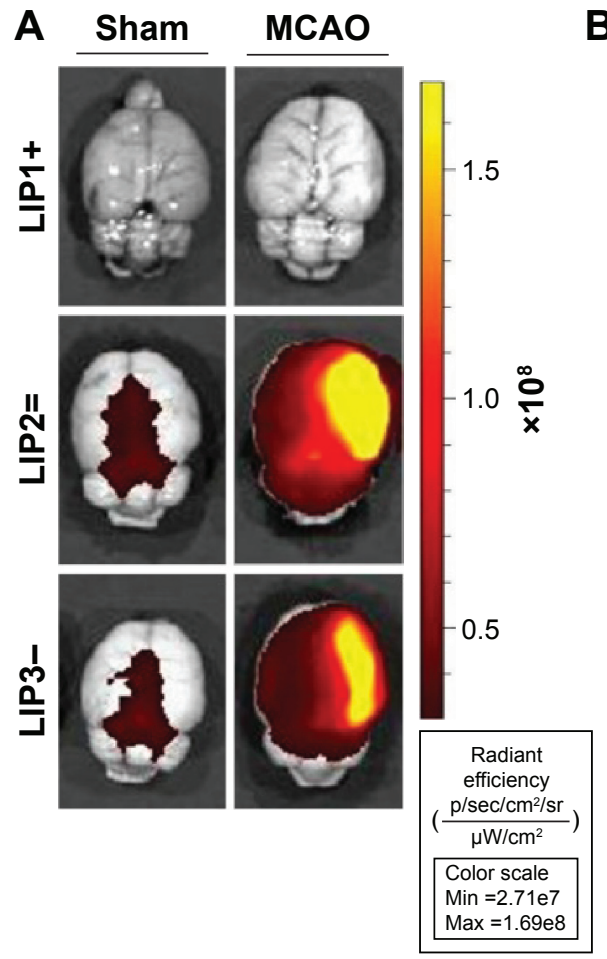

B

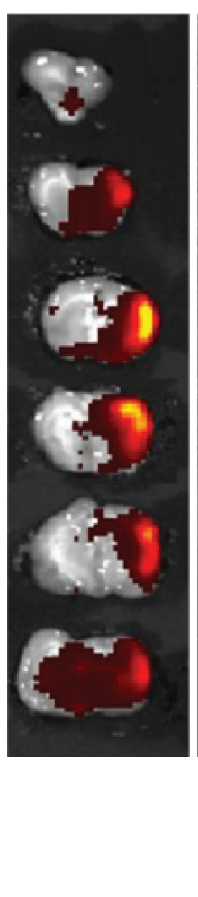

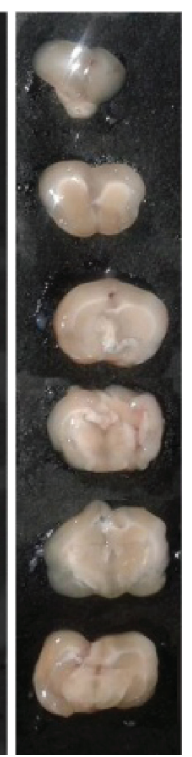

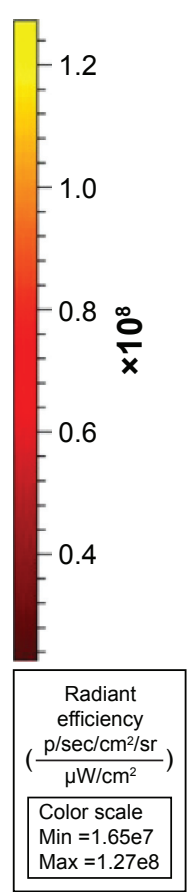

\section{Whole brain liposomes signal}
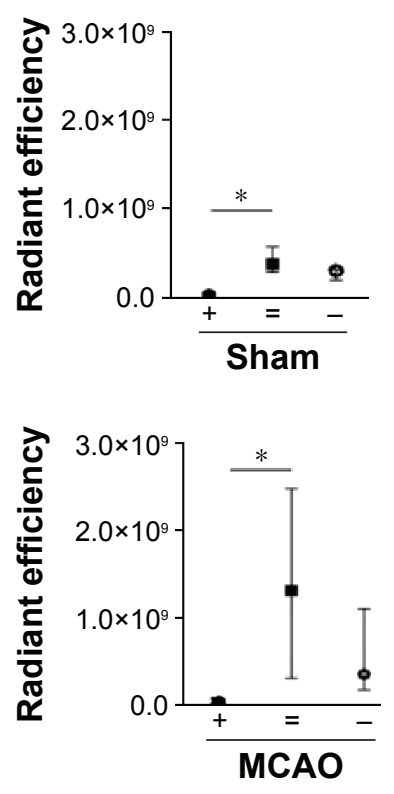

Figure 3 Representative ex vivo rat brain images and fluorescent signal quantifications captured by an imaging system (Xenogen IVIS ${ }^{\circledR}$ Spectrum).

Notes: (A) Whole brain of representative sham and infarcted ischemia rats receiving different net surface charged liposomes. (B) Representative sliced rat brain after being submitted to an MCAO and receiving neutral liposomes. Fluorescent signal colocalized with infarcted area. (C) Quantifications of liposome-emitted signal in sham and MCAO rat brain expressed as radiant efficiency. Images are adjusted to the scale positioned beside. Data present four independent experiments in which three to four animals per group were included. Median values (interquartile range) are represented and significant differences are indicated as $* P<0.05$.

Abbreviations: LIPI+, positive liposomes; LIP2=, neutral liposomes; LIP3-, negative liposomes. MCAO, middle cerebral arterial occlusion; p, photons; sec, seconds; sr, steradian. 
the brain only when compared with the signal after the administration of positive liposomes (neutral: $1.320 \mathrm{e}+009$ [3.040e+008, 2.490e+009] vs positive: $3.430 \mathrm{e}+007$ [5.550e+006, 7.890e+007], $P<0.05$ ) (Figure 3C). Again, negative liposomes presented a higher signal than the positive ones but without reaching statistical significance (negative: $1.78 \mathrm{e}+008[1.78 \mathrm{e}+008,1.10 \mathrm{e}+009])$. Similar quantifications were obtained when brains were sliced into coronal sections (data not shown). Remarkably, when liposomes (neutral or negatively charged) were administered in ischemic animals, signal tended to concentrate in the ischemic hemisphere, mostly in the infarcted region, rather than on the CL side. Ischemic region is observed as a pale area in nonfluorescent brain slices, and it is also known considering the patterns of the animal model used (Figure 3B). ${ }^{22}$

\section{Liposome distribution in other tissues}

Ninety minutes after liposome administration, different organs were analyzed by Xenogen IVIS ${ }^{\circledR}$ Spectrum to explore liposome affinity to different tissues depending on their net surface charge. Liver, spleen, kidneys, and lungs were examined in sham $(\mathrm{n}=11)$ and ischemic animals $(\mathrm{n}=10)$ (Figure 4).
Our results show that only sham rats treated with positive liposomes presented a significant accumulation in the liver compared to the neutral and negative liposomes (positive: $2.012 \mathrm{e}+011 \pm 2.120 \mathrm{e}+010$ vs neutral: $1.393 \mathrm{e}+011 \pm 2.319 \mathrm{e}+010$, $P<0.05$ vs negative: $1.267 \mathrm{e}+011 \pm 2.173 \mathrm{e}+010, P<0.01)$ (Figure 4A). Likewise, only sham rats treated with positive liposomes presented a higher signal in lungs compared to neutral liposomes (positive: 7.230e+009 [6.750e+009, 5.480e+010] vs neutral: $2.865 \mathrm{e}+009$ [5.670e+008, 3.970e+009], $P<0.05$ ), while the comparison with negative liposomes did not reach statistical significance (negative: $3.18 \mathrm{e}+009$ [2.44e+009, 3.6e+009]; Figure 4D). Nonsignificant differences were observed for the differently charged liposomes when spleen and kidneys were evaluated (Figure 4B and C). No comparison among ischemic groups presented significant differences.

\section{Liposome bioavailability in plasma}

Plasma collected 90 minutes after liposome administration exhibited unexpected differences between liposome-charged groups. Sham animals showed a significant longer blood circulation time of neutral liposomes compared with the positive ones (neutral: 6.445e+007 [4.960e+007, 6.900e+007]
A
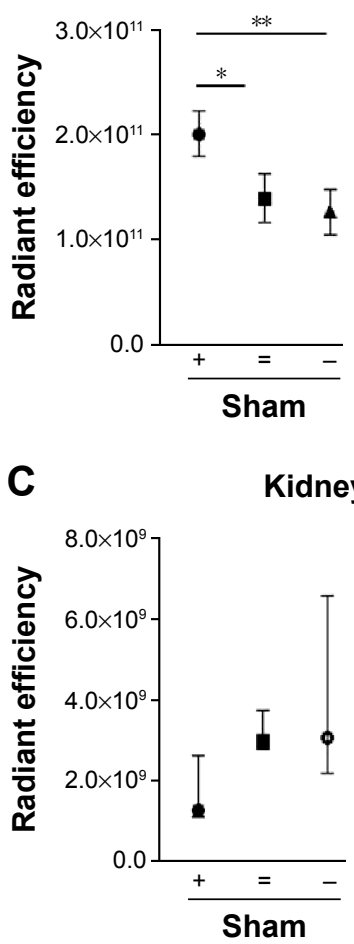

Liver liposomes signal

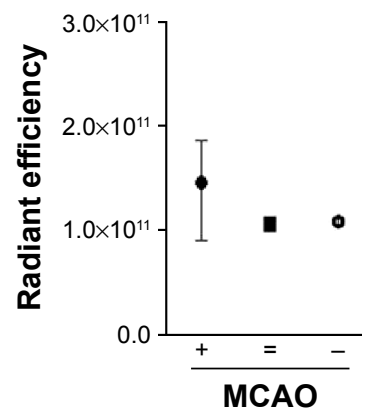

liposomes signal

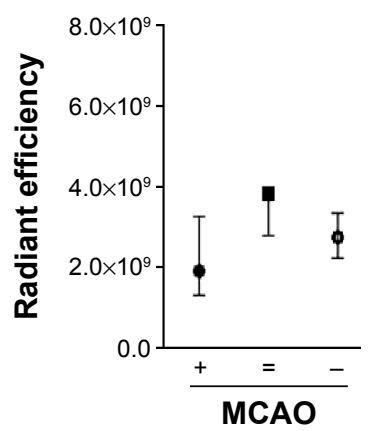

B

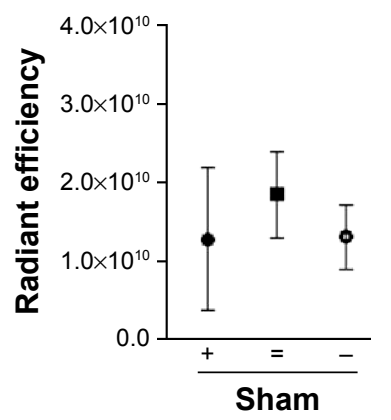

D

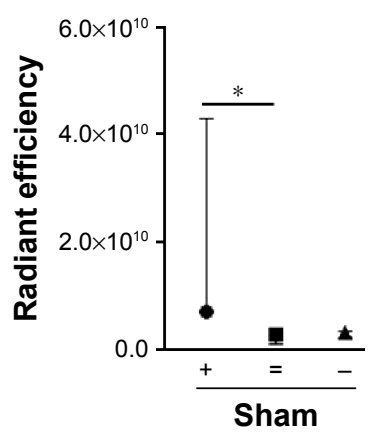

Spleen liposomes signal

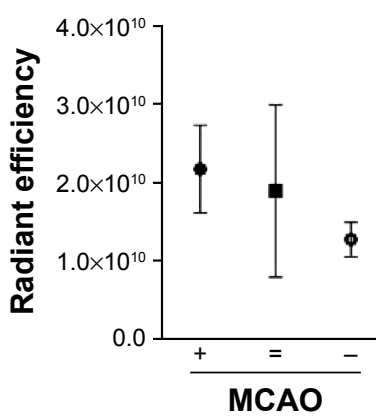

Lung liposomes signal

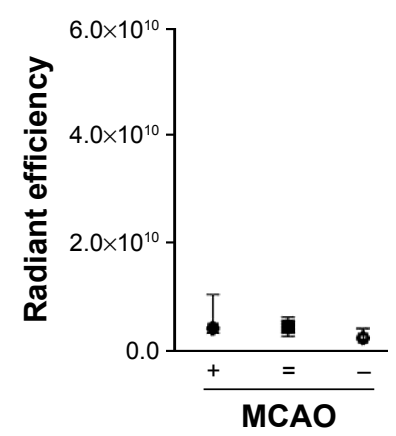

Figure 4 Fluorescent signal quantifications of images captured by Imaging system (Xenogen IVIS ${ }^{\circledR}$ Spectrum) considering distribution of differentially charged liposomes within the body in sham and MCAO rats.

Notes: Liposome accumulation in (A) liver, (B) spleen, (C) kidneys, and (D) lungs. In each group, three to four animals were included. Mean \pm SD or median values (interquartile range) are represented depending on normality data distribution and significant differences are indicated as $* P<0.05, * * P<0.0 \mathrm{I}$.

Abbreviations: +, positive; =, neutral; -, negative; MCAO, middle cerebral arterial occlusion; SD, standard deviation. 
vs positive: $2.115 \mathrm{e}+006[480,000,4.840 \mathrm{e}+006], P<0.05$ ). Likewise, ischemic rats treated with neutral liposomes showed a significant higher fluorescent signal in plasma compared to animals treated with positive liposomes (neutral: $1.100 \mathrm{e}+008[5.210 \mathrm{e}+007,1.250 \mathrm{e}+008]$ vs positive: $3.995 \mathrm{e}+006[1.840 \mathrm{e}+006,6.020 \mathrm{e}+006], P<0.05)$ (Figure 5A and B). Here, although fluorescent signal emitted by neutral liposomes was higher than that emitted by negative liposomes in both sham and ischemic animals, differences did not reach statistical significance (sham negative: $4.15 \mathrm{e}+007$ [1.810e+007, 6.270e+007]; ischemic negative: $2.240 \mathrm{e}+007$ [1.830e+007, 2.840e+007]).

To further study the consequences of administering neutral or negatively charged liposomes, the plasma bioavailability of these two groups was examined in greater detail at different time points. Strikingly, 90 minutes after administration, neutral liposomes exhibited a significantly elevated signal compared to negative liposomes (neutral: $1.437 \mathrm{e}+008 \pm 8.737 \mathrm{e}+006$ vs negative: $6.353 \mathrm{e}+007 \pm 9.278 \mathrm{e}+006, P<0.001)$. Similarly, the signal detected for neutral liposomes was also higher 4 hours after administration (neutral: $3.767 \mathrm{e}+007 \pm 1.386 \mathrm{e}+007$ vs negative: $1.470 \mathrm{e}+007 \pm 4.987 \mathrm{e}+006, P<0.05$ ), whereas hardly any signal was observed at 24 hours in any of the groups (Figure 5C and D).

\section{Simvastatin detection in the brain}

Considering the results of our first substudy, neutral liposomes were selected as the proper candidates for brain delivery. Consequently, in order to study simvastatin arrival into the brain, neutral liposomes were characterized after being loaded with the drug (LIP4S) (Figure 6A). When loaded with simvastatin, neutral liposomes showed a mean diameter of $151.85 \mathrm{~nm}$ (polydispersity index $=0.15$ ) and a neutral surface charge of $-1.01 \mathrm{mV}$. The encapsulation efficiency of simvastatin by liposomes was $64.37 \% \pm 7.55 \%$. By cryogenic transmission electron microscopy analysis, it was also observed that liposomes incorporating the simvastatin still presented the characteristic small multivesicular vesicles (SUV) morphology (Figure 6B).

Here, it is important to mention that by analyzing brain hemisphere homogenates by UHPLC technique, we were able to detect both simvastatin forms (active and inactive), although simvastatin administered to animals was entirely

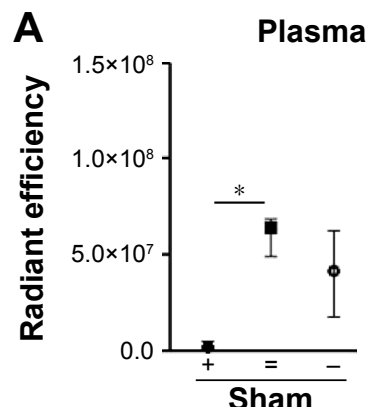

C

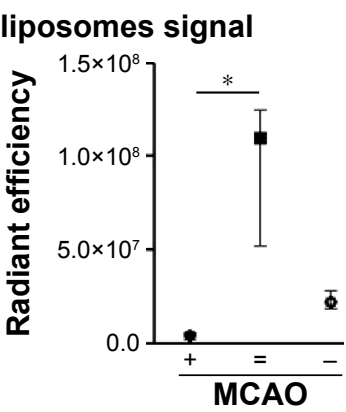

C Liposome kinetics in plasma

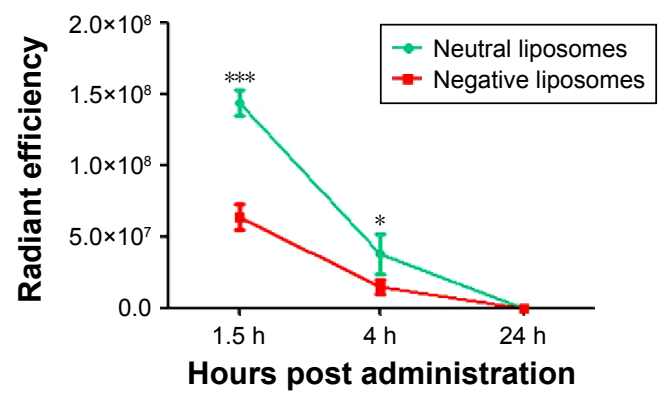

B
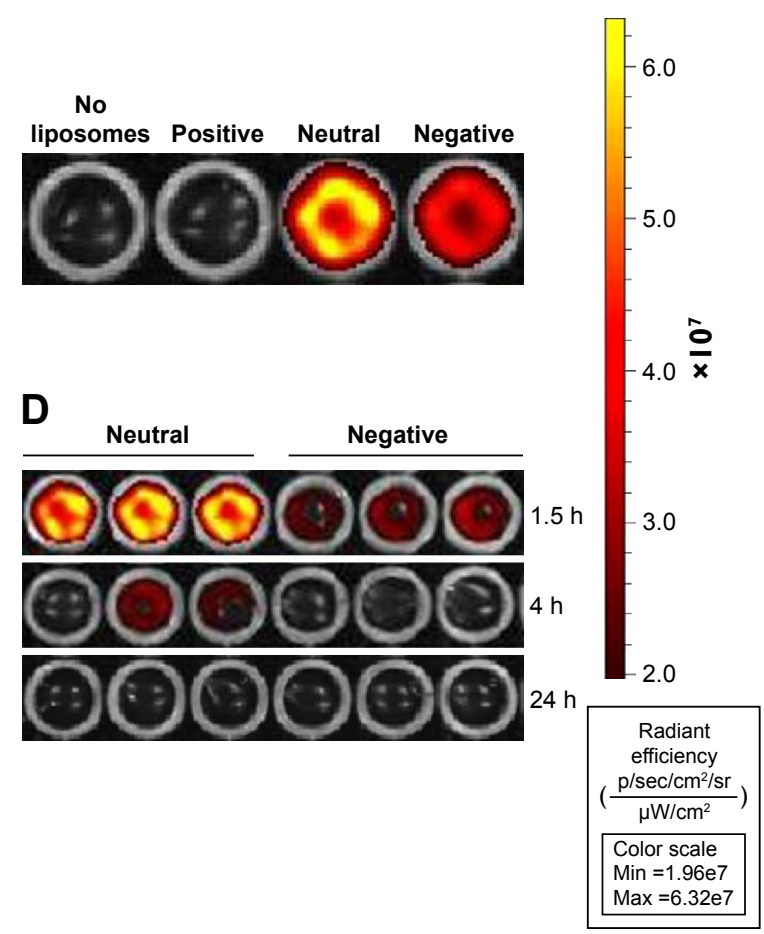

Figure 5 Presence of liposomes in plasma.

Notes: (A) Plasma collected from sham or ischemic rats 90 minutes after receiving liposomes. Median values (interquartile range) are represented and significant differences are indicated as $* P<0.05$. (B) Representative plasma image of one experiment. (C) Liposome kinetics in plasma. Blood was collected from naïve rats (with no surgical procedure) after 1.5 hours, 4 hours, and 24 hours of receiving neutral or negatively charged liposomes. Symbols indicated mean \pm SD. (D) Image representing data on graph (C). Images are adjusted to the scale positioned beside. In all experiments, three to four animals per group were considered. Significant differences are represented as $* P<0.05$, and $* * * P<0.001$.

Abbreviations: +, positive; =, neutral; -, negative; MCAO, middle cerebral arterial occlusion; h, hours; p, photons; sec, seconds; sr, steradian; SD, standard deviation. 
A

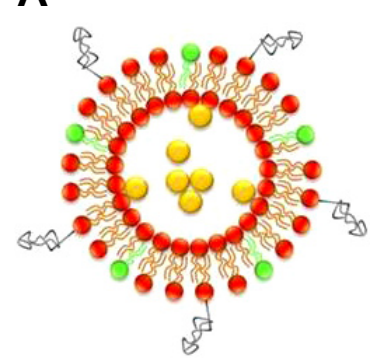

B

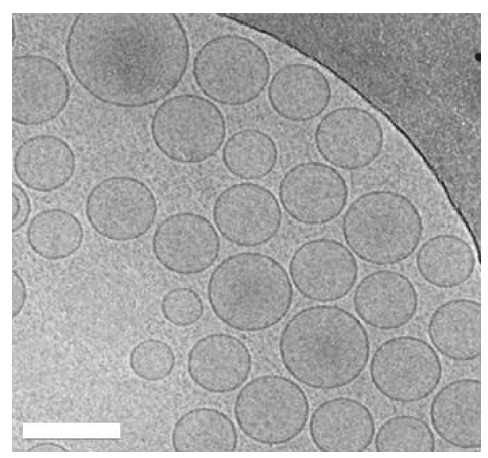

$\mathbf{C}_{100}$

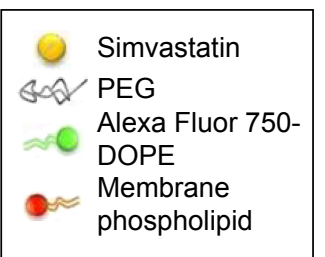

우

0

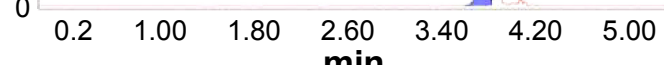

D
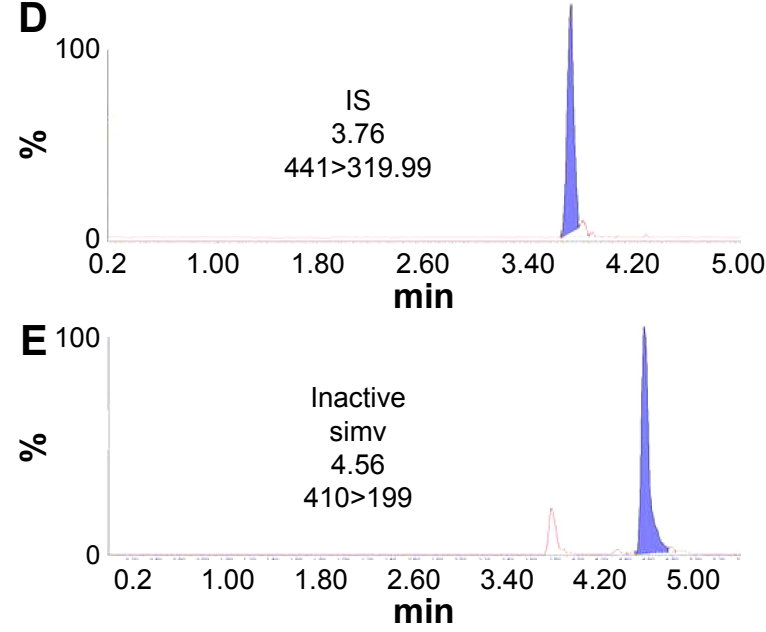

Figure 6 Simvastatin-loaded liposome characterization.

Notes: (A) Structure and composition of neutral liposome compounds used in detection experiments. (B) CryoTEM image of liposomes with simvastatin encapsulated formed by the lipid mixture DLPC/CHOL/CHOL-PEG (scale bar: $200 \mathrm{~nm}$ ). (C-E) UHPLC chromatographic profile of peaks corresponding to (C) active simvastatin (also named acid simvastatin), (D) IS (simvastatin hydroxy acid ammonium salt), and (E) inactive simvastatin (also named lactone). For each compound analyzed, retention time (expressed in minutes) and transition are presented.

Abbreviations: CryoTEM, cryogenic transmission electron microscopy; DLPC, I,2-didodecanoyl-sn-glycero-3-phosphocholine; CHOL, cholesterol; CHOL-PEG, cholesteryl-polyethylene glycol 600 sebacate; UHPLC, ultra-high-protein liquid chromatography; IS, internal standard; DOPE, I,2-dioleoyl-sn-glycero-3-phosphoethanolamine; simv, simvastatin; min, minute; PEG, polyethylene glycol.

activated following the aforementioned protocol ("Liposome formulation and characterization" section).

An IS consisting in simvastatin hydroxy acid ammonium salt was used to correct simvastatin quantification. As shown in Figure $6 \mathrm{C}-\mathrm{E}$, the three peaks corresponding to active simvastatin, IS, and inactive simvastatin, respectively, were easily distinguishable. The three peaks were observed in all analyzed samples.

Through UHPLC technique, we were able to demonstrate that free active simvastatin was able to cross the BBB (Figure 7A), as it was detectable in rat brains 2 hours after administration. In general terms, higher active simvastatin levels were detected when rats were euthanized at 2 hours compared with 4 hours posttreatment. This difference was clearly evidenced when active simvastatin levels were compared between IP ischemic groups (IP ischemic [isch] 2 hours: $2.6[2.3,3.8]$ vs IP isch 4 hours: 0.85 [0.3, 1.4], $P<0.05$ ). Interestingly, ischemic brains showed higher simvastatin accumulation in the IP hemisphere than in the CL hemisphere (2 hours: IP isch: $2.6[2.3,3.8]$ vs CL isch: 1.8 [0.0, 2.4], $P<0.05$ ), whereas no differences were detected between hemispheres in brains from sham animals.
Concerning the detection of free simvastatin in its inactive form (Figure 7B), higher amounts were also obtained after 2 hours compared to 4 hours in the ischemic groups (IP isch 2 hours: $81[66,88]$ vs IP isch 4 hours: $9.6[4.8$, 17.30], $P<0.05$; and CL isch 2 hours: 77.10 [75.50, 85.70] vs $C L$ isch 4 hours: $7.6[4.7,13.30], P<0.05)$. Contrarily, no differences were found between inactive simvastatin in the ischemic areas compared to the CL or sham brains.

We then focused on the comparison between simvastatin levels in the rat brain when it was intravenously administered encapsulated into liposomes or as a free drug. Regarding detection of the active form (Figure 7C), we observed higher levels when it was encapsulated compared with the free form in sham animals, although the difference was significant only at 4 hours post administration (sham free 4 hours: 0.35 $[0.3,0.4]$ vs sham liposomes (lipos) 4 hours: $0.65[0.6,0.9]$, $P<0.05$ ). In contrast, no differences were observed when comparing free and encapsulated administration in ischemic animals at any of the evaluated times (isch free 2 hours: 2.6 $[2.3,4.7]$ vs isch lipos 2 hours: $3.35[1.3,2.4]$ and isch free 4 hours: $0.85[0.3,1.4]$ vs isch lipos 4 hours: $0.9[0.5,1])$. 
A

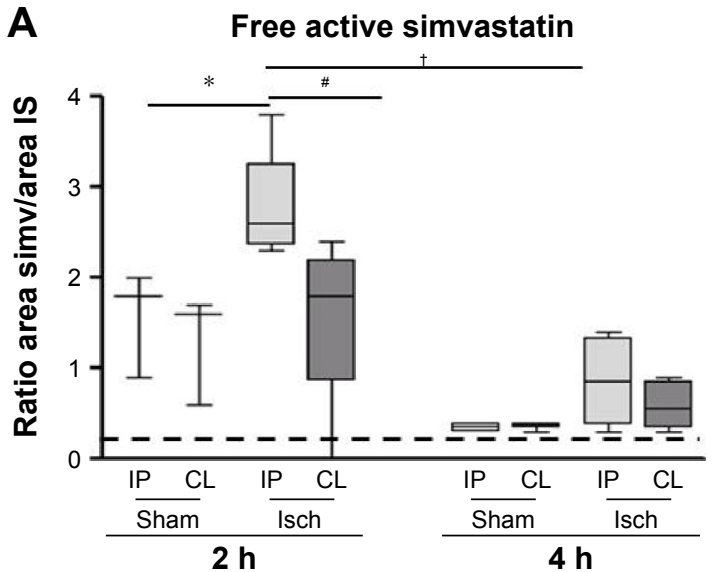

C Active simvastatin in IP hemisphere

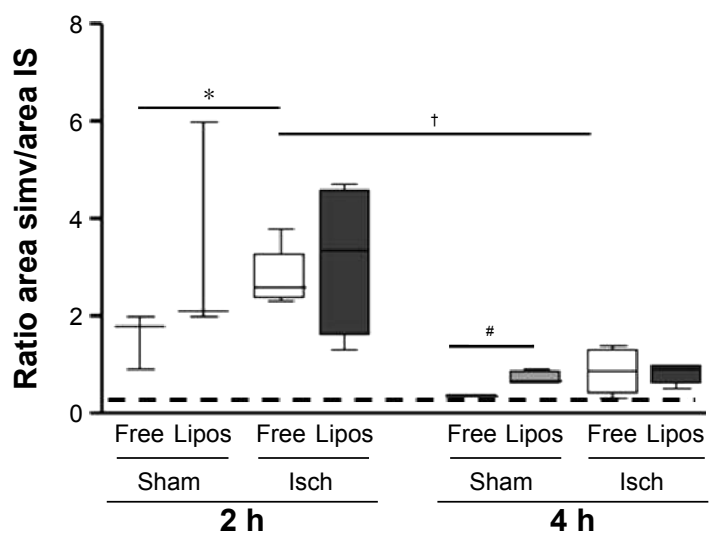

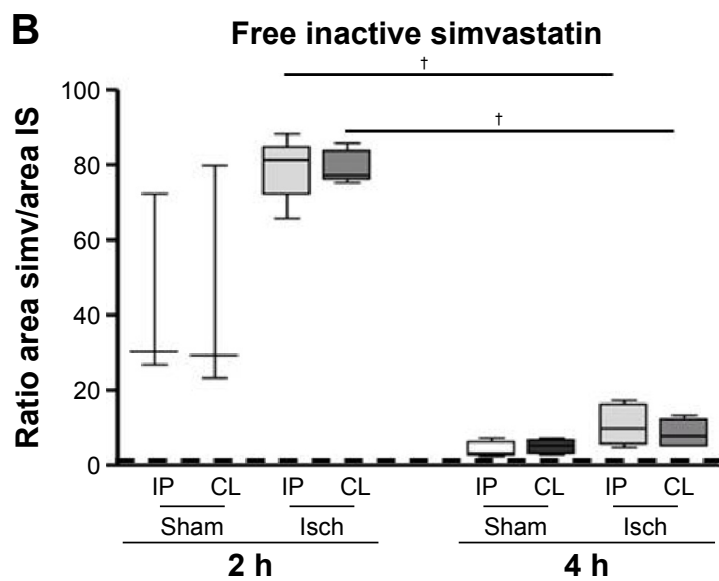

D Inactive simvastatin in IP hemisphere

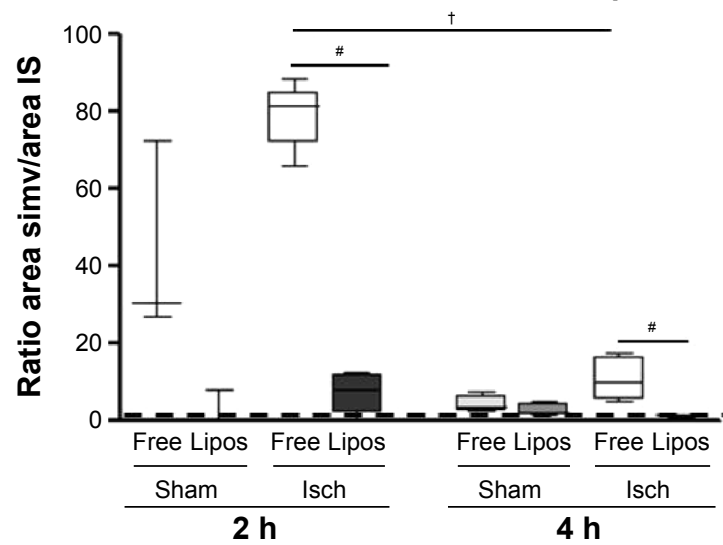

Figure 7 Simvastatin detection in brain tissue through UHPLC technique after intravenous administration of I mL of free simvastatin or simvastatin encapsulated into neutral liposomes.

Notes: All rats were treated with the same simvastatin dose $(20 \mathrm{mg} / \mathrm{kg})$. Rats were submitted to sham or MCAO surgery, treated 90 minutes later, and killed 2 hours or 4 hours after treatment administration. (A) Detection of simvastatin in its active form when rats were treated with free simvastatin and euthanized 2 hours or 4 hours later. (B) Detection of simvastatin in its lactone form (inactive) when rats were treated with free simvastatin and killed 2 hours or 4 hours later. (C) Detection of simvastatin in its active form in IP hemispheres when rats were treated with free or encapsulated simvastatin and killed 2 hours or 4 hours later. (D) Detection of simvastatin in its lactone form (inactive) in IP hemispheres when rats were treated with free or encapsulated simvastatin and killed 2 hours or 4 hours later. In all experiments, three to five animals per group were included. Box plots indicated median (interquartile range). After applying Mann-Whitney test, significant differences are represented as $* P<0.05$ for comparisons between sham and ischemic animals, ${ }^{\#} P<0.05$ for comparisons between IP and CL hemispheres (A) and Free and Lipos treatments (C, D) and $+P<0.05$ for comparisons between 2 hours and 4 hours. Broken lines represent background signal detected through UHPLC technique when brains of naïve animals without receiving treatment were analyzed.

Abbreviations: UHPLC, ultra-high-protein liquid chromatography; MCAO, middle cerebral arterial occlusion; IP, ipsilateral; CL, contralateral; IS, internal standard; simv, simvastatin; h, hours; Isch, ischemic; Lipos, liposomes.

Finally, we evidenced that simvastatin levels in the nonactive form were almost negligible when the drug was encapsulated into liposomes (Figure 7D). Thus, ischemic rats treated with the free form presented significantly higher brain retention of inactive simvastatin at both 2 hours and 4 hours than when it was previously encapsulated (isch free 2 hours: 81.40 [66.0, 88.20] vs isch lipos 2 hours: 7.95 [0.2, 12.40], $P<0.05$, and isch free 4 hours: $9.65[4.8,17.30]$ vs isch lipos 4 hours: $1.2[0.9,1.4], P<0.05)$.

\section{Discussion}

As previously described, the use of a liposomal drug delivery system is a promising strategy to avoid side effects and enhance drug efficiency by changing the distribution of the intact drug. ${ }^{12}$ Liposomes show several advantages, such as biocompatibility or nontoxicity, ability to protect their cargo from degradation by plasma enzymes, and the capacity to transport their load across biological membranes. ${ }^{23}$ To date, liposomes have been used clinically as delivery systems for therapeutic chemotherapeutic agents, antibiotics, and antifungals showing an increase in the safety and efficacy of some drugs. ${ }^{24}$ Taking into account the difficulty for neuroprotectants to reach the target zone due to the BBB characteristics, we considered that the development of a suitable liposomal carrier to encapsulate neuroactive compounds was highly encouraging. 
Aggarwal et $\mathrm{al}^{25}$ previously stated that nanoparticle properties such as size, shape, solubility, surface modifications, and surface charge (zeta potential) can determine their distribution within the body. For instance, the presence of PEG on the surface of liposomes has been shown to extend blood circulation time while reducing mononuclear phagocyte system uptake and consequently decreasing their recognition by the liver and spleen. ${ }^{21,26}$ On the other hand, plasma proteins are described as binding the surface of nanoparticles immediately upon their introduction into a physiological environment, forming a nanoparticle-protein complex that affects the internalization process of nanoparticles into macrophages and their overall distribution throughout the body. ${ }^{25,27,28}$ Accordingly, Gessner et al ${ }^{29}$ postulated plasma protein absorption as a key factor for the in vivo organ distribution of intravenously administered colloidal drug carriers and concluded that this biodistribution was strongly influenced by the surface characteristics of nanoparticles.

In this study, we produced and characterized liposomes with different net surface charges to show how liposome surface charge helps to define their behavior and distribution, supporting the aforementioned hypothesis.

From our data, and in spite of the small sample size of the groups, we can conclude that positively charged liposomes present different behaviors in comparison to neutral or negatively charged ones. Positive liposomes were not detectable in brain tissue, which was likely attributable to different factors. On one hand, they were also absent in plasma 90 minutes after administration, probably due to the high plasma protein absorption. Accordingly, Hernández-Caselles et $\mathrm{a}^{30}$ found a good correlation between the amounts of blood proteins absorbed by liposomes and their stability, the liposomes with the highest protein intake being the most unstable. Furthermore, in another study, cationic particles were found to strongly bind to human erythrocytes, and this fact was reported to lead to the impossibility of liposomes crossing the BBB in the presence of blood cells..$^{31}$ On the other hand, we could detect positively charged liposomes significantly retained in lungs and liver compared with the other two liposomes. In this sense, retention of particles in the lungs consequently impedes their circulation in the blood stream, while Kupffer cells in the liver have been described as playing an important role in also removing liposomes from the circulation, thus accelerating their elimination process. ${ }^{32}$

When we analyzed the liposome detection in brains from sham or ischemic rats, we observed that neutral and negative liposomes were able to cross the BBB, either when its permeability was altered (after MCAO) or when it remained intact (sham animals). Data from sham animals turned out to be quite homogenous, whereas higher intragroup variability was obtained among ischemic rats, probably attributable to the lesion severity.

Furthermore, we demonstrated that negative and neutral liposomes were prone to accumulate in the ischemic core of the brain. In this sense, we consider that the BBB disruption in this area, as well as the inflammatory processes around the ischemic lesion, is responsible for this interesting accumulation, thus agreeing with the statement of Schroeder et al, who consider that the great interest in liposomal delivery systems stems from their ability to accumulate in sites of increased vascular permeability. ${ }^{33-36}$

From our results, we speculate that whereas in sham animals liposome-cell interactions are needed to cross the $\mathrm{BBB}$, liposomes can take advantage of the endothelium damage, a phenomenon usually called "passive targeting", in ischemic animals, leading to a higher local accumulation in the infarcted area. ${ }^{36,37}$

The presence of liposomes in plasma for 4 hours indicated that, without charge, liposomes presented higher bioavailability as their signal was significantly higher compared with the negative and positive particles. Again, as observed in the brain, positive liposomes seemed to undergo a process of degradation, as no fluorescent signal could be detected at 4 hours. Regarding the lack of signal in brain and plasma, showing the inability to cross the BBB or the poor bioavailability, we considered positively charged liposomes as poor candidates to deliver neuroprotectant drugs into the brain and discarded them for further experiments in our study. Additionally, in order to examine whether neutral or negatively charged liposomes were the most proper delivery carriers, a plasma kinetics study was performed, analyzing blood liposome signals from naïve animals at different time points. Neutral liposomes showed a more intense fluorescent signal both at 90 minutes and at 4 hours after being administered. Concordantly, some in vitro studies have demonstrated that neutral particles have slower opsonization rates than charged particles, thus preventing their phagocytosis and contributing to a prolonged presence in plasma. ${ }^{28,38}$

Taken as a whole, several factors seem to affect liposome distribution, and their surface charge is undoubtedly a determinant to be taken into consideration in future therapeutic strategies. However, regarding the controversial literature previously published in which particle cationization is considered a way to improve the ability of particles to cross the BBB, we support other authors who concluded that the type of lipid mixture was also a key feature that can affect the 
rate of liposome clearance. ${ }^{24,39}$ Our hypothesis is that the vast possibilities of liposome fabrication and the infinite drugs that can take advantage of its encapsulation make it really difficult to draw comparisons between previously published studies. Nevertheless, we reached the same conclusion as relevant systematic studies that have evaluated the pharmacokinetics and biodistribution of charged PEGylated liposomes: negative particles exhibit strong reticuloendothelial system uptake; positive particles induce serum protein aggregation and neutral nanoparticles exhibit the least reticuloendothelial system interaction and the longest circulation, being the most suitable ones. ${ }^{40}$

The experiments on simvastatin analysis in rat brain after IV administration corroborated its ability to cross the BBB per se, as other authors previously stated. ${ }^{41,42}$ Simvastatin is lipophilic in nature and therefore easily diffuses across enterocytes' cell membranes. Interestingly, the highest accumulation was observed in infarcted hemispheres of ischemic animals, probably due to BBB alteration after ischemia. As other studies reported, with the BBB being weakened, systemically administered drugs can undergo enhanced extravasation rates in the cerebral endothelium, leading to increased parenchymal drug concentrations. ${ }^{43}$ Moreover, we could detect more simvastatin in the rat brain at 2 hours than at 4 hours after being administered. It is reasonable that the drug concentration decreases while being in the tissue, and it is also in agreement with Bellosta et $\mathrm{al},{ }^{44}$ who reported simvastatin elimination half-life ( $t_{1 / 2}$ [hours]) as $2-3$ hours. On the other hand, it should be emphasized that simvastatin is an inactive hydrophobic lactone prodrug, which is commonly administered orally. After oral administration, it is described as being metabolized in vivo to several more polar and pharmacologically active compounds by both liver and plasma esterases. ${ }^{45-47}$ Given that some stroke patients suffer from dysphagia, we consider that parenteral statin administration would represent an improvement for their clinical management. This is the reason why, in our study, simvastatin was activated before its encapsulation and/or intravenously administered. Our aim was to optimize the amount of active simvastatin and consequently its effect. Strikingly, but in accordance with others, we observed that simvastatin metabolism is reversible and that interconversion occurs between the lactone (inactive) and acid (active) forms. ${ }^{45,48}$ Consequently, we detected both forms when analyzing brain samples through UHPLC technique.

Remarkably, simvastatin encapsulation into liposomes exhibited some unexpected results. First, and contrarily to our hypothesis, we did not detect higher active simvastatin accumulation in ischemic brain region after being encapsulated into liposomes. Second, sham animals presented more simvastatin accumulation when administered in liposomes. Third, liposomes seem to prevent simvastatin deactivation, as animals treated with encapsulated simvastatin presented very low levels of the inactive form.

Although our data showed similar levels of active simvastatin in ischemic areas after free or encapsulated simvastatin administration, thus not demonstrating an enhancement in drug delivery occurred, we could state that liposomes do not impede simvastatin brain penetration. In this regard, variability among infarct sizes and small sample size could have negatively interfered with our data. At this point, we assume the impossibility of calculating infarct volumes as a clear limitation of the study. Correlation between infarct severity and liposome accumulation in brain would undoubtedly be of high interest.

On the other hand, data obtained from sham animals are highly remarkable. We observed that when encapsulated, simvastatin can be easily retained in brain tissue compared with free simvastatin. This could become an important improvement for those human patients, who receive simvastatin as a preventive treatment. A higher simvastatin brain accumulation could enable a dose reduction and, consequently, fewer possibilities to suffer secondary effects.

Finally, the effect of liposomes on altering simvastatin interconversion from active to inactive form would also represent a clear advantage. In this sense, Skottheim et $\mathrm{a}^{49}$ reported that simvastatin lactone showed 37-fold higher potential to induce myotoxicity compared to its acid forms in a study with human skeletal muscle cells in vitro. Furthermore, a remarkable increase in metabolic clearance was noted in another study, for all statin lactones compared with their acid forms.$^{48}$ In accordance with these results, Romana et $\mathrm{al}^{11}$ reported that nanodelivery systems such as liposomes might solve statin problems including adverse effects on muscles and liver or their poor bioavailability. As we were able to demonstrate that liposomes can accumulate in the infarct area and also that simvastatin can be delivered after being encapsulated, further in vivo studies are needed in order to explore the neuroprotection effect of encapsulated simvastatin, as well as other attributable beneficial effects such as liver or muscle toxicity reduction.

\section{Conclusion}

This study confirms that liposome charge is critical to promote its accumulation in the brain infarct area after transient MCAO. Furthermore, encapsulation into neutral liposomes does not 
hamper simvastatin from being delivered in brain tissue. Thus, simvastatin encapsulation might be a promising strategy to ensure that the drug reaches the brain, while increasing its bioavailability and reducing possible side effects.

\section{Acknowledgments}

We are grateful to Yolanda Fernández Amurgo and Anna Pujol Esclusa for their excellent technical support with ex vivo imaging experiments. The research leading to these results received funding from the European Union's Seventh Framework Program (FP7/2007-2013) under grant agreements number 201024 and number 202213 (European Stroke Network). Neurovascular Research Laboratory takes part in the Spanish stroke research network INVICTUS (RD12/0014/0005). This study was partially funded by projects FIS 11/0176 on stroke biomarkers research and EC07/90195 on increasing safety and efficacy of simvastatin neuroprotection. Simvastatin was a donation from Grupo Uriach, Barcelona, Spain.

\section{Disclosure}

$\mathrm{MC}-\mathrm{M}$ is supported by a predoctoral fellowship grant (FI 10/00508), and MH-G and AR are senior researchers in the Miguel Servet Program (CP12/03259 and CP09/00265), all three from the Instituto de Salud Carlos III of the Spanish Ministry of Economy. AS is supported by a predoctoral fellowship (2015 FI_B 00952) from the Agencia de Gestió d'Ajuts Universitaris i de Recerca de Catalunya. The authors report no other conflicts of interest in this work.

\section{References}

1. Roger VL, Go AS, Lloyd-Jones DM, et al; American Heart Association Statistics Committee and Stroke Statistics Subcommittee. Executive summary: heart disease and stroke statistics - 2012 update: a report from the American Heart Association. Circulation. 2012;125(1):188-197.

2. Kwiatkowski TG, Libman RB, Frankel M, et al. Effects of tissue plasminogen activator for acute ischemic stroke at one year. National Institute of Neurological Disorders and Stroke Recombinant Tissue Plasminogen Activator Stroke Study Group. N Engl J Med. 1999;340(23): 1781-1787.

3. García-Bonilla L, Campos M, Giralt D, et al. Evidence for the efficacy of statins in animal stroke models: a meta-analysis. J Neurochem. 2012; 122(2):233-243.

4. Campos-Martorell M, Salvador N, Monge M, et al. Brain proteomics identifies potential simvastatin targets in acute phase of stroke in a rat embolic model. J Neurochem. 2014;130(2):301-312.

5. Jonsson N, Asplund K. Does pretreatment with statins improve clinical outcome after stroke? A pilot case-referent study. Stroke. 2001; 32(5):1112-1115.

6. Martí-Fàbregas J, Gomis M, Arboix A, et al. Favorable outcome of ischemic stroke in patients pretreated with statins. Stroke. 2004;35(5):1117-1121.

7. Balduini W, De Angelis V, Mazzoni E, Cimino M. Simvastatin protects against long-lasting behavioral and morphological consequences of neonatal hypoxic/ischemic brain injury. Stroke. 2001;32(9):2185-2191.

8. Jain MK, Ridker PM. Anti-inflammatory effects of statins: clinical evidence and basic mechanisms. Nat Rev Drug Discov. 2005;4(12):977-987.
9. Wang C-Y, Liu P-Y, Liao JK. Pleiotropic effects of statin therapy: molecular mechanisms and clinical results. Trends Mol Med. 2008; 14(1):37-44.

10. Tiwari G, Tiwari R, Sriwastawa B, et al. Drug delivery systems: an updated review. Int J Pharm Investig. 2012;2(1):2-11.

11. Romana B, Batger M, Prestidge CA, Colombo G, Sonvico F. Expanding the therapeutic potential of statins by means of nanotechnology enabled drug delivery systems. Curr Top Med Chem. 2014;14(9):1182-1193.

12. Ishii T, Fukuta T, Agato $Y$, et al. Nanoparticles accumulate in ischemic core and penumbra region even when cerebral perfusion is reduced. Biochem Biophys Res Commun. 2013;430(4):1201-1205.

13. Sahagun G, Moore SA, Hart MN. Permeability of neutral vs. anionic dextrans in cultured brain microvascular endothelium. Am J Physiol. 1990; 259(1 pt 2):H162-H166.

14. García-Bonilla L, Sosti V, Campos M, et al. Effects of acute posttreatment with dipyridamole in a rat model of focal cerebral ischemia. Brain Res. 2011;1373:211-220.

15. Pérez-Asensio FJ, Hurtado O, Burguete MC, et al. Inhibition of iNOS activity by $1400 \mathrm{~W}$ decreases glutamate release and ameliorates stroke outcome after experimental ischemia. Neurobiol Dis. 2005; 18(2):375-384.

16. Banes-Berceli AK, Shaw S, Ma G, et al. Effect of simvastatin on high glucose- and angiotensin II-induced activation of the JAK/STAT pathway in mesangial cells. Am J Physiol Renal Physiol. 2006;291(1): F116-F121.

17. Lowery A, Onishko H, Hallahan DE, Han Z. Tumor-targeted delivery of liposome-encapsulated doxorubicin by use of a peptide that selectively binds to irradiated tumors. J Control Release. 2011;72(2):181-204.

18. Afergan E, Ben David M, Epstein H, et al. Liposomal simvastatin attenuates neointimal hyperplasia in rats. AAPS J. 2010;12(2):181-187.

19. Papagiannaros A, Kale A, Levchenko TS, Mongayt D, Hartner WC, Torchilin VP. Near infrared planar tumor imaging and quantification using nanosized Alexa 750-labeled phospholipid micelles. Int J Nanomedicine. 2009;4:123-131.

20. Zhang J, Rodila R, Gage E, et al. High-throughput salting-out assisted liquid/liquid extraction with acetonitrile for the simultaneous determination of simvastatin and simvastatin acid in human plasma with liquid chromatography. Anal Chim Acta. 2010;661(2):167-172.

21. Immordino ML, Dosio F, Cattel L. Stealth liposomes: review of the basic science, rationale, and clinical applications, existing and potential. Int J Nanomedicine. 2006;1(3):297-315.

22. Joo SP, Xie W, Xiong X, Xu B, Zhao H. Ischemic postconditioning protects against focal cerebral ischemia by inhibiting brain inflammation while attenuating peripheral lymphopenia in mice. Neuroscience. 2013;243:149-157.

23. Elbayoumi TA, Torchilin VP. Current trends in liposome research. Methods Mol Biol. 2010;605:1-27.

24. Spuch C, Navarro C. Liposomes for targeted delivery of active agents against neurodegenerative diseases (Alzheimer's disease and Parkinson's disease). J Drug Deliv. 2011;2011:469679.

25. Aggarwal P, Hall JB, McLeland CB, Dobrovolskaia MA, McNeil SE. Nanoparticle interaction with plasma proteins as it relates to particle biodistribution, biocompatibility and therapeutic efficacy. Adv Drug Deliv Rev. 2009;61(6):428-437.

26. Kobeissy FH, Ottens AK, Zhang Z, et al. Novel differential neuroproteomics analysis of traumatic brain injury in rats. Mol Cell Proteomics. 2006;5(10):1887-1898.

27. Moghimi SM, Hunter AC, Murray JC. Long-circulating and targetspecific nanoparticles: theory to practice. Pharmacol Rev. 2001;53(2): 283-318.

28. Owens DE, Peppas NA. Opsonization, biodistribution, and pharmacokinetics of polymeric nanoparticles. Int J Pharm. 2006;307(1):93-102.

29. Gessner A, Lieske A, Paulke B, Müller R. Influence of surface charge density on protein adsorption on polymeric nanoparticles: analysis by two-dimensional electrophoresis. Eur J Pharm Biopharm. 2002; 54(2):165-170. 
30. Hernández-Caselles T, Villalaín J, Gómez-Fernández JC. Influence of liposome charge and composition on their interaction with human blood serum proteins. Mol Cell Biochem. 1993;120(2):119-126.

31. Weiss L, Zeigel R, Jung OS, Bross ID. Binding of positively charged particles to glutaraldehyde-fixed human erythrocytes. Exp Cell Res. 1972;70(1):57-64.

32. Swaroop Kumar K, Jyothi T, Nagendar S, Devipriya S, Vijaya Kumar G, Sudhakar Babu AM. Stability of liposomes. Pak J Pharm Sci. 1995; 8(2):69-79.

33. Kuroiwa T, Ting P, Martinez H, Klatzo I. The biphasic opening of the blood-brain barrier to proteins following temporary middle cerebral artery occlusion. Acta Neuropathol. 1985;68(2):122-129.

34. Belayev L, Busto R, Zhao W, Ginsberg MD. Quantitative evaluation of blood-brain barrier permeability following middle cerebral artery occlusion in rats. Brain Res. 1996;739(1-2):88-96.

35. Yuan F, Leunig M, Huang SK, Berk DA, Papahadjopoulos D, Jain RK. Microvascular permeability and interstitial penetration of sterically stabilized (stealth) liposomes in a human tumor xenograft. Cancer Res. 1994;54(13):3352-3356.

36. Schroeder A, Turjeman K, Schroeder JE, Leibergall M, Barenholz Y. Using liposomes to target infection and inflammation induced by foreign body injuries or medical implants. Expert Opin Drug Deliv. 2010; 7(10):1175-1189.

37. Lu W. Adsorptive-mediated brain delivery systems. Curr Pharm Biotechnol. 2012;13(12):2340-2348.

38. Roser M, Fischer D, Kissel T. Surface-modified biodegradable albumin nano- and microspheres. II: effect of surface charges on in vitro phagocytosis and biodistribution in rats. Eur J Pharm Biopharm. 1998; 46(3):255-263.

39. Cavaletti G, Cassetti A, Canta A, et al. Cationic liposomes target sites of acute neuroinflammation in experimental autoimmune encephalomyelitis. Mol Pharm. 2009;6(5):1363-1370.
40. Ernsting MJ, Murakami M, Roy A, Li S-D. Factors controlling the pharmacokinetics, biodistribution and intratumoral penetration of nanoparticles. J Control Release. 2013;172(3):782-794.

41. Vuletic S, Riekse RG, Marcovina SM, Peskind ER, Hazzard WR, Albers JJ. Statins of different brain penetrability differentially affect CSF PLTP activity. Dement Geriatr Cogn Disord. 2006;22(5-6):392-398.

42. Saheki A, Terasaki T, Tamai I, Tsuji A. In vivo and in vitro blood-brain barrier transport of 3-hydroxy-3-methylglutaryl coenzyme A (HMG-CoA) reductase inhibitors. Pharm Res. 1994;11(2):305-311.

43. Misra A, Ganesh S, Shahiwala A, Shah SP. Drug delivery to the central nervous system: a review. J Pharm Pharm Sci. 2003;6(2):252-273.

44. Bellosta S, Paoletti R, Corsini A. Safety of statins: focus on clinical pharmacokinetics and drug interactions. Circulation. 2004;109(23 suppl 1): III50-III57.

45. Vickers S, Duncan CA, Chen IW, Rosegay A, Duggan DE. Metabolic disposition studies on simvastatin, a cholesterol-lowering prodrug. Drug Metab Dispos. 1990;18(2):138-145.

46. Mauro VF. Clinical pharmacokinetics and practical applications of simvastatin. Clin Pharmacokinet. 1993;24(3):195-202.

47. Lippert J, Brosch M, von Kampen O, et al. A mechanistic, model-based approach to safety assessment in clinical development. CPT Pharmacometrics Syst Pharmacol. 2012;1:e13.

48. Fujino H, Saito T, Tsunenari Y, Kojima J, Sakaeda T. Metabolic properties of the acid and lactone forms of HMG-CoA reductase inhibitors. Xenobiotica. 2004;34(11-12):961-971.

49. Skottheim IB, Gedde-Dahl A, Hejazifar S, Hoel K, Asberg A. Statin induced myotoxicity: the lactone forms are more potent than the acid forms in human skeletal muscle cells in vitro. Eur J Pharm Sci. 2008;33(4-5):317-325.
International Journal of Nanomedicine

\section{Publish your work in this journal}

The International Journal of Nanomedicine is an international, peerreviewed journal focusing on the application of nanotechnology in diagnostics, therapeutics, and drug delivery systems throughout the biomedical field. This journal is indexed on PubMed Central, MedLine, CAS, SciSearch $®$, Current Contents ${ }^{\circledR} /$ Clinical Medicine,

\section{Dovepress}

Journal Citation Reports/Science Edition, EMBase, Scopus and the Elsevier Bibliographic databases. The manuscript management system is completely online and includes a very quick and fair peer-review system, which is all easy to use. Visit http://www.dovepress.com/ testimonials.php to read real quotes from published authors. 